\title{
Development of Multi-sensor Array Electrodes for Measurement of Deeper Muscle Activation
}

\author{
Yasuharu Koike, ${ }^{1 *}$ Yeongdae Kim, ${ }^{2}$ Sorawit Stapornchaisit, ${ }^{2}$ \\ Zixuan Qin, ${ }^{2}$ Toshihiro Kawase, ${ }^{1}$ and Natsue Yoshimura, ${ }^{1,3}$ \\ ${ }^{1}$ Institute of Innovative Research, Tokyo Institute of Technology, \\ 4259 Nagatsuta-cho, Midori-ku, Yokohama, Kanagawa 226-8502, Japan \\ ${ }^{2}$ Department of Information Processing, Tokyo Institute of Technology, \\ 4259 Nagatsuta-cho, Midori-ku, Yokohama, Kanagawa 226-8502, Japan \\ ${ }^{3}$ PRESTO, JST, 4-1-8, Honcho, Kawaguchi-shi, Saitama 332-0012, Japan
}

(Received September 27, 2019; accepted December 17, 2019)

Keywords: EMG, multi-sensor array electrode, musculoskeletal model, ICA

Muscles of the forearm are related to finger motion, and the forearm is divided into the anterior and posterior compartments. The anterior compartment of the forearm is further divided into the superficial, intermediate, and deep layers, and the posterior compartment into the superficial and deep layers. The superficial-layer muscles are related to wrist motion, and the deep-layer muscles to finger motion. Owing to these anatomical features, electromyogram signals from the superficial layer are mixed with those from the deep layer. In this paper, we introduce a system of multi-sensor array electrodes covering the entire forearm. We developed them to estimate muscle activities from not only the superficial layer but also the deep layer. The electrodes were made from conductive fibers and easy to set up. The EMG signals detected by these sensors were examined by independent component analysis.

\section{Introduction}

When we manipulate objects with our hands, arm posture is properly controlled according to our intention. Our arm has redundant joints, and the posture of the arm is also controlled depending on the task requirement. Depending on the object weight or required force, not only the posture but also force and impedance are controlled simultaneously. To measure these parameters, 3D position, force-torque sensors are used. However, the impedance of joints is difficult to measure because it cannot be measured under a static condition. Perturbation is usually applied to measuring impedance.

Hand motion is realized by muscle contraction, whose force can be estimated by measuring electromyogram (EMG) signals. Muscle contraction pulls the bone and cannot push the bone back to its original position, so an antagonist muscle is required. On the basis of this mechanism, there are an infinite number of combinations of various activities of muscles to generate the same net joint torque. Shin et al. measured several EMG signals from the shoulder

*Corresponding author: e-mail: koike@pi.titech.ac.jp https://doi.org/10.18494/SAM.2020.2636 
and elbow and estimated joint torque from only EMG signals using a musculoskeletal model. ${ }^{(1)}$ In their study, zero net joint torques with different cocontraction levels were also estimated. Kawase et al. applied this model to control a prosthetic hand or a power-assisted robot. ${ }^{(2)}$ They also used electroencephalogram (EEG) signals to estimate EMG signals to control robots. ${ }^{(3)}$

Surface EMG signals are measured to estimate muscle force, and each electrode is attached to a location for a specific muscle. The location of the sensor is quite important and there are several limitations. The activity of a muscle corresponds to the amplitude of the measured signal. The fibers in the middle of a muscle have a larger diameter. When the electrode is located on the innervation zone, the amplitude of EMG signals decreases because of the cancellation of the action potentials of signals traveling in the opposite direction. There is also crosstalk with signals from adjacent muscles.

Muscles of the forearm are related to finger motion, and the forearm is divided into the anterior and posterior compartments. The anterior compartment of the forearm is further divided into the superficial, intermediate, and deep layers, and the posterior compartment into the superficial and deep layers. The superficial-layer muscles are related to wrist motion, and the deep-layer muscles to finger motion. Owing to these anatomical features, EMG signals from the superficial layer are mixed with those from the deep layer.

For estimating muscle fiber conduction velocity, the location of EMG electrodes is quite important and anatomical knowledge is required for the correct placement of the electrodes and to find the innervation points. Linear electrode arrays are being developed. ${ }^{(4,5)}$ Some studies used high-density EMG array electrodes ${ }^{(6,7)}$ to estimate hand kinematics. High-density and two-dimensional grid electrodes have also been developed for finding the localization and size estimation of motor units. ${ }^{(8)}$ The size of an electrode should fit one muscle, so that the electrode can detect the characteristics of each muscle.

In this paper, we introduce a system of multi-sensor array electrodes (MSAEs) covering the forearm to estimate muscle activities from not only the superficial layer but also the deep layer. Independent component analysis (ICA) of multichannel signals was carried out and we found that calculated independent components (ICs) are related to the muscle activities depending on the task of the fingers or wrist.

\section{Materials and Methods}

\subsection{Torque estimation from EMG signals}

Figure 1 shows a schematic image of hand force estimation from EMG signals. Muscle force is estimated on the basis of the musculoskeletal system, and joint torque is calculated from muscle force and moment arm. We have been developing a musculoskeletal model, and using this model, joint torque was estimated from only EMG signals.

$$
\begin{gathered}
f_{\text {muscle }_{j}}=\left(k_{0}+k_{1} u_{j}\right) \times\left(l_{o}+l_{1} u_{j}-\sum a_{i j} \theta_{i}\right) \\
\tau_{i}=\sum_{i} a_{i j} \times f_{\text {muscle }_{j}}
\end{gathered}
$$




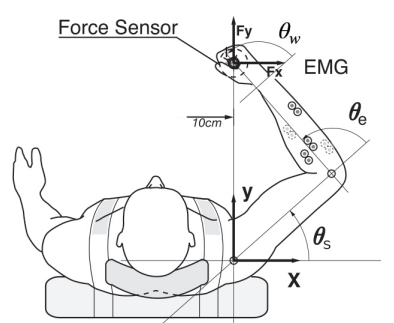

(a)

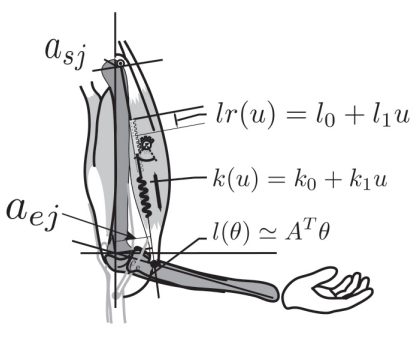

(b)

$$
\begin{aligned}
F & =\left(J(\theta)^{T}\right)^{-1} \tau \\
\tau_{j} & =\sum_{i} a_{i j} f_{\text {muscle }}(\theta, u)
\end{aligned}
$$

(c)

Fig. 1. Signal flows for hand force estimation. (a) Measurement from EMG signals. (b) Muscle force and joint torque estimation. (c) Hard force.

Here $u, i$, and $j$ are the normalized surface EMG signals, the number of the joint, and the number of the muscle, respectively. $f_{\text {muscle }}, k_{0}+k_{1} u_{j}$, and $l_{0}+l_{1} u_{j}$ are the muscle force, the muscle stiffness, and the change in the natural length of the muscle, respectively. $a_{i j}$ is the moment arm, which is the length from the line of muscle to the joint center, and $\theta_{j}$ is the joint angle. Joint torque is calculated from muscle force and moment arm as shown in Fig. 1, and joint stiffness is defined by $\partial \tau / \partial \theta$. The equilibrium angle is calculated using Eq. (1). ${ }^{(1)}$ To apply this musculoskeletal model, each muscle force has to be estimated from sensor signals.

Figure 2 shows (a) a cross section of the forearm, (b) the locations and names of muscles, and (c) the relationship between EMG signals and electrodes. An adjacent electrode measures the EMG signal from the same superficial-layer muscle (red signals), but several electrodes receive signals from the deep-layer muscle (green signals). These red and green signals are measured simultaneously for each electrode.

\subsection{ICA for processing measured signals of deep muscle activation}

ICA is a signal processing method to separate independent signals that are linearly mixed in multiple sensors. ${ }^{(9,10)}$ The problem of blind signal separation is solved by ICA in various research fields, such as speech recognition, data communication, and sensor signal processing.

$$
\begin{aligned}
& \boldsymbol{x}(t)=\boldsymbol{A s}(t), \\
& \boldsymbol{y}(t)=\boldsymbol{W} \boldsymbol{x}(t),
\end{aligned}
$$

where $\boldsymbol{A}$ is an unknown mixing matrix, and $\boldsymbol{s}(t)$ and $\boldsymbol{x}(t)$ are the source signals and observations, respectively. It is difficult to recover the original signals $\boldsymbol{s}(t)$, but the matrix $\boldsymbol{W}$ may recover the original signals to minimize the error between $\boldsymbol{s}(t)$ and $\boldsymbol{y}(t)$.

\subsection{Experimental setup}

One subject participated in the experiment. The study protocol was approved by the ethics committee of the Tokyo Institute of Technology and was carried out in accordance with the Declaration of Helsinki. Written informed consent was obtained from the participant. 


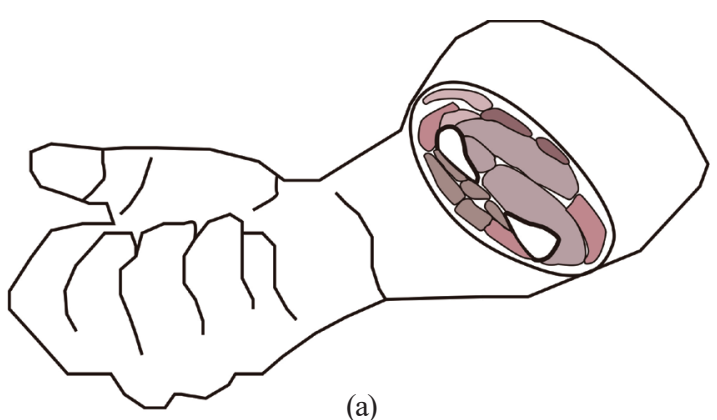

(a)

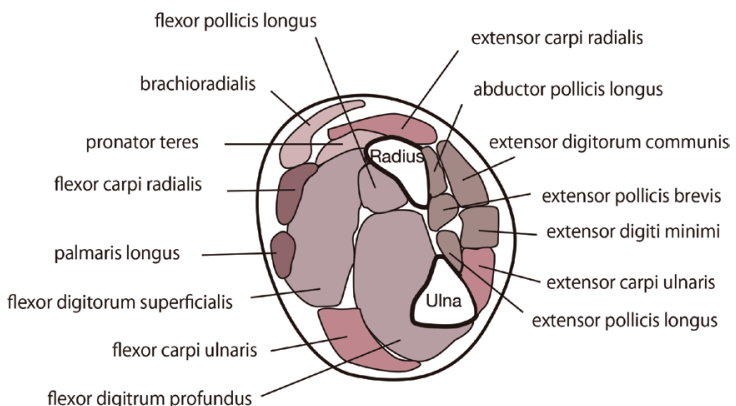

(b)

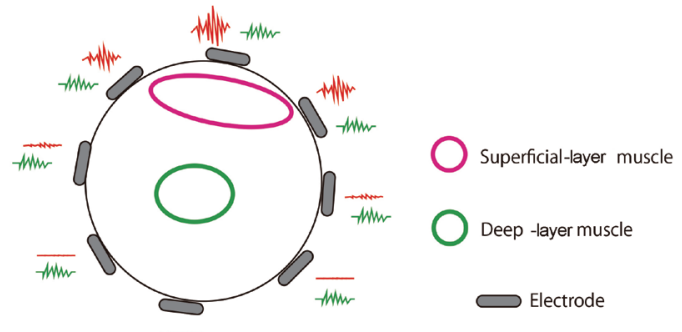

(c)

Fig. 2. (Color online) Muscle locations. (a) Cross section of forearm and (b) muscle locations and names, and (c) relationship between EMG signals and electrodes.

The participant sat on a chair and was given a command by a stimulus program on the screen located $0.5 \mathrm{~m}$ away to perform wrist flexion/extension and finger motion tasks in front of a camera, as shown in Fig. 3. The MSAEs were attached to the right forearm of the participant. EMG signals were measured using our newly developed system of MSAEs (SMK Corp., SEIREN Co. Ltd.). EMG signals were recorded from 32 positions with 24-bit resolution. Signals were acquired at a sampling rate of $500 \mathrm{~Hz}$. EMG data were analyzed by MATLAB, and FastICA was used for ICA components. Wrist and finger motions were measured by an OptiTrack system (NaturalPoint, Inc.).

\section{Results}

Figure 4 shows the raw EMG (blue) and quasi-tension (orange) data from the MSAEs for the flexion/extension task. The flexor muscle and extensor muscle are activated alternately. Some muscles are activated in both the flexion and extension phases. 


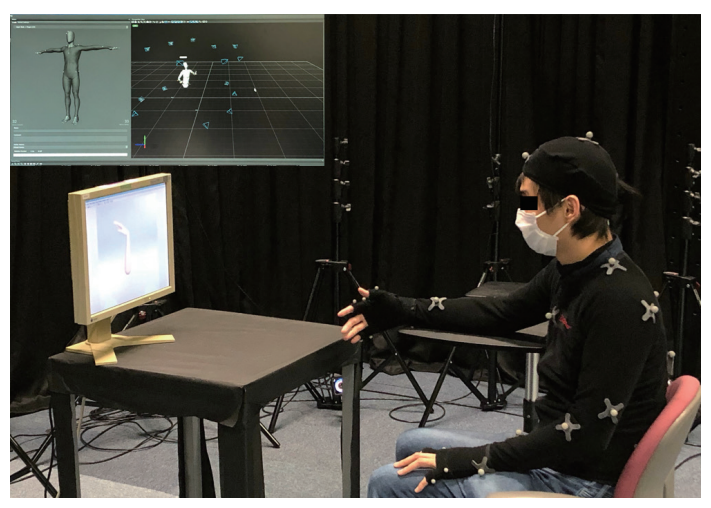

Fig. 3. (Color online) Experimental setup.

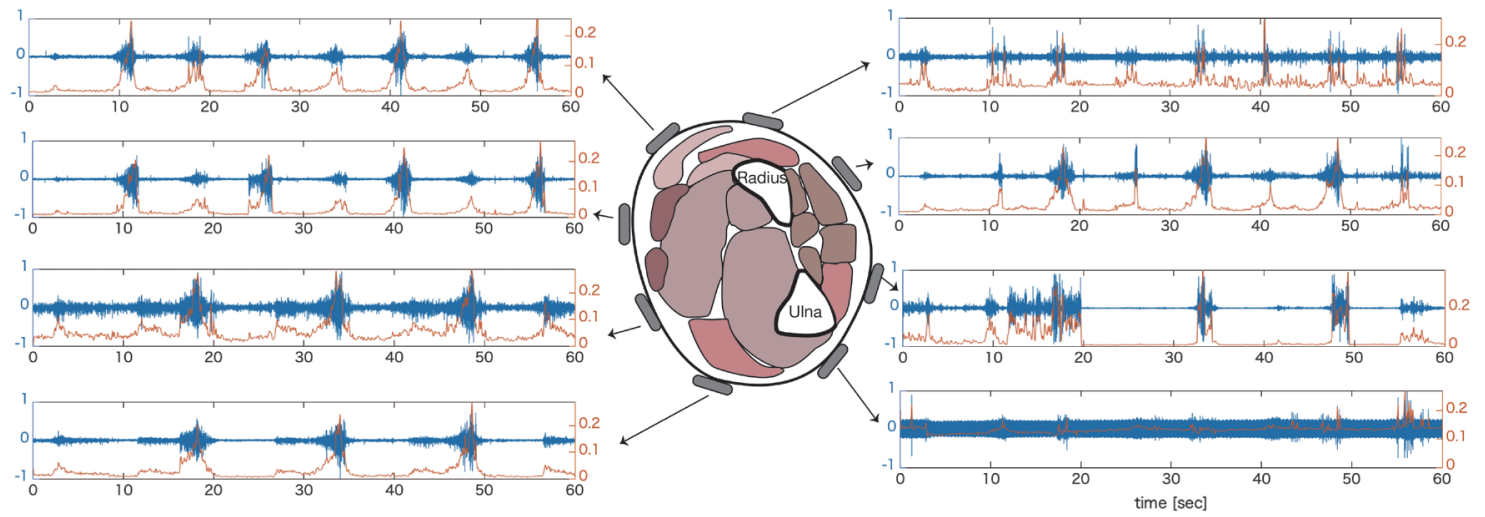

Fig. 4. (Color online) EMG measurement results from MSAEs.

Figure 5(a) shows the joint angle and quasi-tension generated during the task and Fig. 5(b) shows ICA components obtained using one-line sensor data.

The first IC corresponds to the wrist flexion motion and the second IC corresponds to the extension motion. Channels 17 to 23 include an activation pattern similar to that of the first IC, but the second IC eliminates this activation pattern and only the extension activation pattern remains.

Channels 17 to 23 include a similar activation pattern. This means that the signal source is the deep layer.

\section{Discussion}

We showed that the ICA components can be calculated from the data of MSAEs that cover all the muscles in the forearm. From the analysis of the weight matrix $\boldsymbol{W}$ in Eq. (2), the MSAEs were found to be related to the ICA components, as shown in Fig. 6. In each row, a small number of values are large; this means that the signal source is close to an electrode. When many electrodes are related to an ICA component, the signal source is the deep layer, as shown in Fig. 2. 

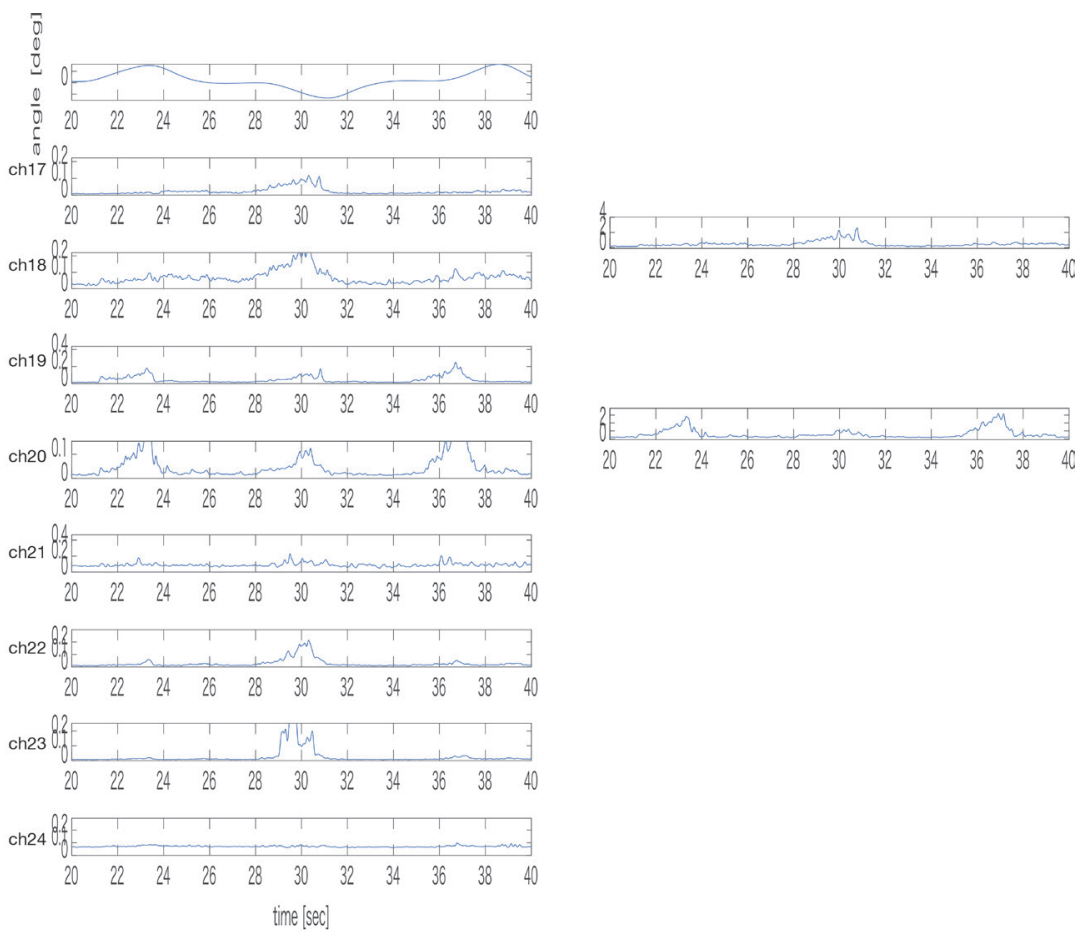

(a)

(b)

Fig. 5. (Color online) (a) EMG sensor data and (b) ICA components.

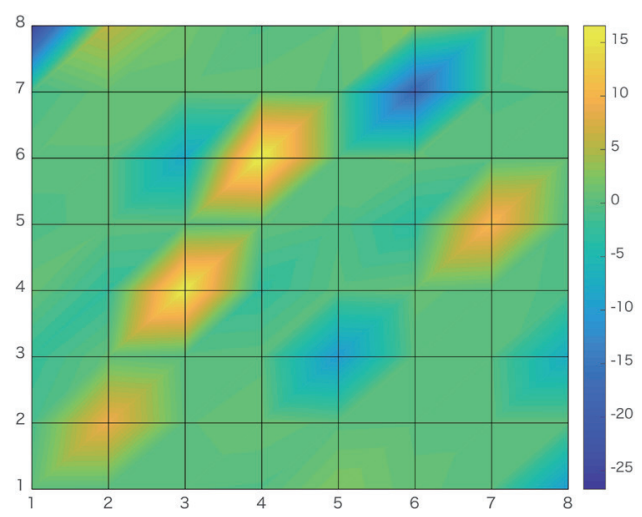

Fig. 6. (Color online) ICA weight values.

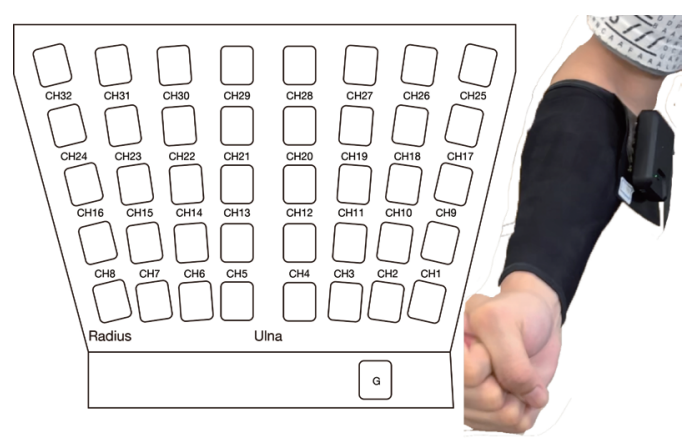

Fig. 7. (Color online) Array electrodes made from conductive fibers.

\section{Conclusions}

In this paper, a system of MSAEs covering the entire forearm to estimate muscle activities from not only the superficial layer but also the deep layer is introduced. The conventional method of placing one electrode at a muscle position is difficult to use in daily life. Recently, conductive fibers have been used for electrodes for measuring heart rate. ${ }^{(11)}$ In collaboration with SMK Corp and SEIREN Co. Ltd., we have been developing a device whose electrodes are made from a conductive fiber and are evenly arranged. Thirty-two channel signals are sent to a PC via Bluetooth (Fig. 7). 
Finger motions were estimated using these signals on the basis of the musculoskeletal model, and ICA showed significantly good results compared with EMG sensor data. ${ }^{(12)}$ Our MSAEs are markedly advantageous for the measurement of EMG signals without any anatomical knowledge about the locations of muscles. However, it took a long time to attach all electrodes. When a user wears this device like a sleeve, the electrodes are not exactly located on the muscles, but the information of EMG activities can be extracted by ICA. In future, the preparation period for measurements will be reduced and the device will be made easy to use.

\section{Acknowledgments}

This work was supported by JSPS under KAKENHI grant number 17H05903, Tateishi Science and Technology Foundation (grant number 2188001), JST PRESTO (Precursory Research for Embryonic Science and Technology), (grant number JPMJPR17JA), and JST MIRAI (grant number JY300171).

\section{References}

1 D. Shin, J. Kim, and Y. Koike: J. Neurophysiol. 101 (2009) 387. https://doi.org/10.1152/jn.00584.2007

2 T. Kawase, N. Yoshimura, H. Kambara, and Y. Koike: Adv. Rob. 31 (2017) 88. https://doi.org/10.1080/0169186 4.2016.121593

3 T. Kawase, H. Kambara, and Y. Koike: J. Rob. Mechatron. 24 (2012) 205.

4 R. Merletti, R. D. Farina, and M. Gazzoni: J. Electromyogr. and Kinesiol. 13 (2003) 37. https://doi.org/10.1016/ S1050-6411(02)00082-2

5 T. Masuda, H. Miyano, and T. Sadoyama: Electroencephalogr. Clin. Neurophysiol. 60 (1985) 435. https://doi. org/10.1016/0013-4694(85)91018-1

6 S. Muceli and D. Farina: IEEE Trans. Neural Syst. Rehabil. Eng. 20 (2012) 371. https://doi.org/10.1109/ TNSRE.2011.2178039

7 S. Muceli, N. Jiang, and D. Farina: IEEE Trans. Neural Sys. Rehab. Eng. 22 (2014) 623. https://doi.org/10.1109/ TNSRE.2013.2282898

8 T. Masuda and C. J. De Luca: J. Electromyogr. Kinesiol. 1 (1991) 116. https://doi.org/10.1016/10506411(91)90005-P

9 S.-I. Amari, A. Cichocki, and H. H. Yang: Adv. Neural Inf. Process. Syst. 8 (1996) 757. Retrieved from https:// papers.nips.cc/paper/1115-a-new-learning-algorithm-for-blind-signal-separation.pdf

10 S. N. Jain: Int. J. Eng. Sci. Technol. 4 (2012) 1490.

11 S. Tsukada, H. Nakashima, and K. Torimitsu: PLoS One 7 (2012). https://doi.org/10.1371/journal.pone.0033689

12 S. Stapornchaisit, Y. Kim, A. Takagi, N. Yoshimura, and Y. Koike: Front. Neurorob. 13 (2019). https:// doi:10.3389/fnbot.2019.00075

\section{About the Authors}

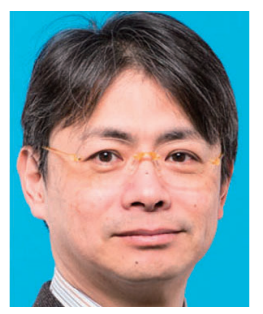

Yasuharu Koike received his B.S., M.S., and Dr. Eng. degrees from Tokyo Institute of Technology, Tokyo, Japan in 1987, 1989, and 1996, respectively. From 1989 to 1998, he worked at Toyota Motor Corporation. From 1991 to 1994, he was at Advanced Telecommunications Research (ATR) in Kyoto, Japan. In 1998, he moved to the Precision and Intelligence Laboratory, Tokyo Institute of Technology, Tokyo, Japan, where he is currently a professor of the Institute of Innovative Research. (koike@pi.titech.ac.jp) 


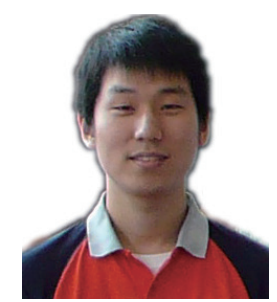

Yeongdae Kim received his B.S. degree from Hangdong University Korea in 2015 and his M.S. degree from Tokyo Institute of Technology, Tokyo, Japan in 2017. He is currently a Ph.D. student at Tokyo Institute of Technology. His research interests include rehabilitation robotics and biological signal processing. (kim.y.ah@m.titech.ac.jp)

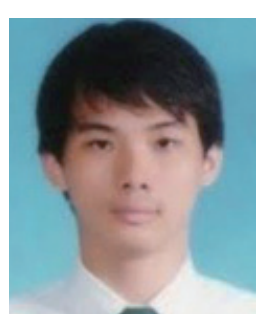

Sorawit Stapornchaisit received his B.S. and M.S. degrees from Kasetsart University, Thailand in 2013 and 2015, respectively, and his M.S. degree from Tokyo Institute of Technology, Tokyo, Japan in 2017. He is currently a Ph.D. student at Tokyo Institute of Technology. His research interests include robotics and biological signal processing. (stapornchaisit.s.aa@m.titech.ac.jp)

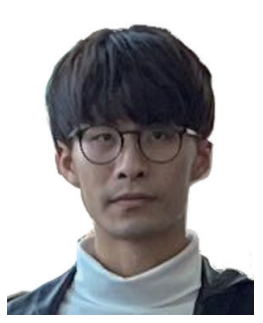

Zixuan Qin received his B.S. degree from Dalian University of Technology, China in 2018. He is currently a master's student at Tokyo Institute of Technology. His research interests include rehabilitation robotics and biological signal processing. (qin.z.aa@m.titech.ac.jp)

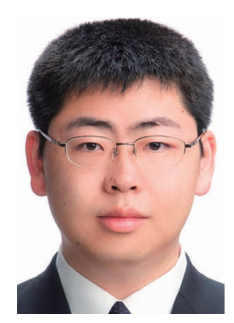

Toshihiro Kawase received his B.S., M.S., and Ph.D. degrees from Tokyo Institute of Technology, Tokyo, Japan in 2007, 2009, and 2012, respectively. $\mathrm{He}$ is currently an adjunct assistant professor at Tokyo Institute of Technology. His research interests include rehabilitation robotics and biological signal processing. (kawase.t.aa@m.titech.ac.jp)

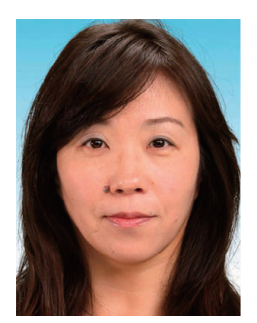

Natsue Yoshimura received her M.S. degree from Tokyo Medical and Dental University, Japan in 2006, and her Ph.D. degree from The University of Electro-communications, Japan in 2009. She was a postdoctoral researcher at Tokyo Institute of Technology from 2009 to 2010 and became an assistant professor. She has been an associate professor at the Institute of Innovative Research, Tokyo Institute of Technology, since 2015. Her research interests include brain machine/computer interfaces. (yoshimura@pi.titech.ac.jp) 